\title{
Hautkrebs in der Hausarztpraxis: Häufigkeit, Fortbildungsbedarf und subjektive Diagnosesicherheit. Eine Umfrage unter Zürcher Hausärzten
}

Badertscher, Nina ; Senn, Oliver ; Rossi, Pascal O ; Wensing, Michel ; Rosemann, Thomas ; Tandjung, Ryan

\begin{abstract}
Background: Incidence of malignant skin lesions is rising in Switzerland. We wanted to assess the frequency of patients with suspicious skin lesions in daily practice. Furthermore, we asked for diagnostic certainty and the need of further education in skin cancer. Methods: Survey with 1212 GPs in the canton of Zurich, descriptive analysis, regression analysis. Results: Response rate 41,4\%. 23,2\% of the GPs saw daily/weekly patients with suspicious skin lesions, another 34,8\% monthly. 79,0\% reported need for further education in skin cancer. This need was lower in urban GPs and in GPs with higher diagnostic certainty. Conclusions: GPs saw relatively often patients with suspicious skin lesions. Despite high diagnostic certainty, there was a clear need for further education in skin cancer.
\end{abstract}

DOI: https://doi.org/10.1024/1661-8157/a001466

Other titles: Skin cancer in primary care: frequency, need for further education and subjective diagnostic certainty. A cross sectional survey among general practitioners in the canton of Zurich

Posted at the Zurich Open Repository and Archive, University of Zurich ZORA URL: https://doi.org/10.5167/uzh-84298

Journal Article

Accepted Version

Originally published at:

Badertscher, Nina; Senn, Oliver; Rossi, Pascal O; Wensing, Michel; Rosemann, Thomas; Tandjung, Ryan (2013). Hautkrebs in der Hausarztpraxis: Häufigkeit, Fortbildungsbedarf und subjektive Diagnosesicherheit. Eine Umfrage unter Zürcher Hausärzten. Praxis, 102(22):1353-1359.

DOI: https://doi.org/10.1024/1661-8157/a001466 


\section{Institut für Hausarztmedizin, Universität Zürich}

Hautkrebs in der Hausarztpraxis: Häufigkeit, Fortbildungsbedarf und subjektive Diagnosesicherheit.

Eine Umfrage unter Zürcher Hausärzten.

Skin Cancer in Primary Care: Frequency, Need for Further Education and Subjective Diagnostic Certainty.

A Cross Sectional Survey Among General Practitioners in the Canton of Zurich.

Nina Badertscher ${ }^{1,3}$, Oliver Senn ${ }^{1}$, Pascal O. Rossi ${ }^{1}$, Michel Wensing ${ }^{2}$, Thomas Rosemann ${ }^{1}$, Ryan Tandjung ${ }^{1}$

${ }^{1}$ Institut für Hausarztmedizin, Universität Zürich

${ }^{2}$ Radboud University Nijmegen Medical Centre, The Netherlands

${ }^{3}$ Korrespondierende Autorin:

Dr. med. Nina Badertscher

Institut für Hausarztmedizin

Pestalozzistrasse 24

8091 Zürich

Email: nina.badertscher@usz.ch

Datum: 08.05.2013 


\section{Abstract:}

Background: Incidence of malignant skin lesions is rising in Switzerland. We wanted to assess the frequency of patients with suspicious skin lesions in daily practice. Furthermore, we asked for diagnostic certainty and the need of further education in skin cancer.

Methods: Survey with 1212 GPs in the canton of Zurich, descriptive analysis, regression analysis.

Results: Response rate $41.4 \%$. $23.2 \%$ of the GPs saw daily/weekly patients with suspicious skin lesions, another $34.8 \%$ monthly. $79.0 \%$ reported need for further education in skin cancer. This need was lower in urban GPs and in GPs with higher diagnostic certainty.

Conclusions: GPs saw relatively often patients with suspicious skin lesions. Despite high diagnostic certainty, there was a clear need for further education in skin cancer.

Key words: primary care, general practitioner, dermatology, skin lesion, skin cancer 


\section{Zusammenfassung:}

\section{Einleitung:}

Die Inzidenz maligner Hautveränderungen steigt in der Schweiz kontinuierlich. Ziel unserer Umfrage war es, zu ermitteln, wie häufig Hausärzte Patienten mit malignomverdächtigen Hautveränderungen sehen, wie sicher sie in der Diagnose sind und ob Fortbildungsbedarf zum Thema Hautkrebs besteht.

\section{Material/ Methoden:}

Umfrage bei 1212 Hausärzten im Kanton Zürich. Deskriptive Auswertung, Untersuchung von Einflussfaktoren auf den Fortbildungsbedarf mittels Regressionsanalysen.

\section{Resultate:}

41.4\% Rücklauf. 23.2\% aller Hausärzte sahen täglich/wöchentlich Patienten mit malignomverdächtigen Hautläsionen, weitere $34.8 \%$ sahen monatlich entsprechende Patienten. 79.0\% der Hausärzte gaben Fortbildungsbedarf an, dieser war geringer bei städtischen Hausärzten und bei hoher subjektiver Diagnosesicherheit.

\section{Schlussfolgerung:}

Zürcher Hausärzte wurden relativ häufig mit malignomverdächtigen Hautveränderungen ihrer Patienten konfrontiert. Zudem bestand trotz relativ hoher subjektiver Diagnosesicherheit ein deutlicher Fortbildungsbedarf zum Thema Hautkrebs.

Schlüsselwörter: Hausarztmedizin, Hausarzt, Hautkrebs, Dermatologie, Hautläsion 


\section{Ü1 Einleitung}

In der Schweiz ist Hautkrebs eine der häufigsten Krebsarten überhaupt (1). In den letzten Jahren hat die Häufigkeit von Hautkrebs bereits markant zugenommen und ein weiterer Anstieg wird erwartet (2-4). Aktuell erkranken pro Jahr ca. 1900 Personen an einem Melanom (1) und für im Jahr 2000 Geborene beträgt das Lebenszeitrisiko, an einem Melanom zu erkranken bereits 1:80 (2). Im europaweiten Vergleich belegt die Schweiz bezüglich Melanom-Prävalenz einen der vordersten Plätze (5). Gründe hierfür können bekannte Hautkrebs-Risikofaktoren wie heller Hauttyp mit Neigung zu dysplastischen Nävi, familiäre Belastung sowie chronische Sonnenschäden sein (6-9). Auch (für die Schweizer Bevölkerung relativ typische) freizeit- oder ferienbedingte intermittierend hohe UV-Belastung in ansonsten eher sonnenungewohnten Populationen stellt einen Risikofaktor dar und erklärt, warum höheres Einkommen mit einem erhöhten Hautkrebsrisiko vergesellschaftet ist (10).

Die drei wichtigsten Formen von Hautkrebs sind das Basalzellkarzinom, das spinozelluläre Karzinom und das Melanom. Das Melanom ist die aggressivste Form von Hautkrebs. Wird es nicht früh genug erkannt und korrekt behandelt, besteht die grosse Gefahr der Metastasen-Entwicklung. Sind solche Metastasen einmal vorhanden, ist das Melanom meist nicht mehr heilbar und führt oft innerhalb kurzer Zeit zum Tod. Das Basalzellkarzinom und das spinozelluläre Karzinom bilden extrem selten Metastasen, führen auf Grund ihres lokal destruktiven Wachstums und ihrer Rezidivneigung aber zu erheblicher Morbidität und zu hohen Gesundheitskosten (11).

Die zunehmende Inzidenz, verbunden mit erhöhter Sensibilisierung der Bevölkerung für Hautkrebs, führt dazu, dass Hausärzte immer häufiger als erste Anlaufstelle für Patienten mit verdächtigen Hautläsionen fungieren. Um die Betreuung dieser Patienten in den Hausarztpraxen sicherzustellen, ist es wichtig, dass die Hausärzte sich im Bereich Hautkrebs auf hohem Niveau fortbilden können. Das Institut für Hausarztmedizin Zürich untersucht in Zusammenarbeit mit der Dermatologischen Klinik des Universitätsspitals Zürich im Rahmen einer Studie einen neuen Fortbildungsansatz für Hausärzte, der die Technik der Teledermatologie mit dem Prinzip der kontinuierlichen Schulung verbindet (Projekt „minSKIN“) (12). Ziel der hier präsentierten Umfrage war es, im Vorfeld der minSKIN-Studie herauszufinden, ob bei den Hausärzten im Kanton Zürich Fortbildungsbedarf zum Thema HautkrebsDiagnostik besteht. Zudem fragten wir die Hausärzte unter anderem nach Häufigkeit des Themas in ihrer täglichen Praxis sowie nach ihrer subjektiv wahrgenommenen Diagnosesicherheit bei malignomverdächtigen Hautveränderungen. 


\section{Ü1 Material und Methoden}

Für diese Umfrage wurde ein Kurzfragebogen zum Thema „Hautkrebs in der Hausarztpraxis“ entwickelt, der nebst Angabe einiger demographischer Daten und einer Freitext-Frage sechs Einfachauswahlfragen sowie eine Mehrfachauswahlfrage enthielt. Es wurden unter anderem Fragen gestellt zur Häufigkeit des Themas Hautkrebs in der Hausarztpraxis, zur subjektiven Beurteilungssicherheit, zum Fortbildungsbedarf diesbezüglich und zum konkreten Vorgehen bei Patienten mit malignomverdächtigen Hautveränderungen.

„Pilotierungsphase“: Der Fragebogen wurde im Januar 2011 an die rund 60 Teilnehmer eines Lehrärztetreffens des Instituts für Hausarztmedizin Zürich abgegeben. Der Fragebogen musste aufgrund der Rückmeldungen von 39 Fragebogen aus der Pilotierungsphase nicht angepasst werden, da die Fragen als klar und relevant eingeschätzt wurden.

Definitiver Versand: Im März 2011 wurde der Fragebogen erfolgreich an 1212 Hausärzte im Kanton Zürich verschickt, wobei die Lehrärzte des Treffens diesen Versand nicht erneut erhielten, um eine doppelte Datenerhebung zu verhindern. Dem Fragebogen wurde ein vorfrankiertes Rückantwortcouvert beigelegt. Bei fehlender Antwort wurde nach 6 Wochen der Fragebogen zusammen mit einem Erinnerungsschreiben erneut zugestellt.

Sowohl die Fragebogen aus der Pilotierungsphase wie auch die Fragebogen aus dem definitiven Versand wurden anonym ausgewertet.

Der Praxisstandort wurde folgendermassen klassifiziert: Anhand der Postleitzahlen der Praxisstandorte wurde ermittelt, ob in der entsprechenden Ortschaft mindestens ein Dermatologe praktizierte. Wenn dies der Fall war, wurde die Praxis als „städtisch“ klassifiziert, ansonsten als „ländlich“.

Für die statistischen Analysen wurde das Statistikprogramm STATA, Version 12 verwendet. In einem ersten Schritt wurden die Daten deskriptiv ausgewertet. In einem zweiten Schritt wurde die Korrelation zwischen möglichen Einflussfaktoren auf den dermatologischen Fortbildungsbedarf der befragten Hausärzte mittels Spearmans Korrelationskoeffizienten analysiert. Aufgrund multipler Vergleiche wurden die Korrelationsanalysen mittels Bonferroni Korrektur angepasst. Die Assoziation zwischen Einflussfaktoren und dem dermatologischen Fortbildungsbedarf wurde mittels bivariater 
und multipler logistischer Regression untersucht. Für diese Analysen wurde die unabhängige Variable „Fortbildungsbedarf“ dichotomisiert (ja/eher ja versus nein). Auch für den Faktor "Häufigkeit“ wurden die Antworten dichotomisiert (täglich/wöchentlich versus monatlich/seltener). Ebenso wurde für den Faktor „Sicherheit“ verfahren (eher sicher/sehr sicher versus eher unsicher/sehr unsicher). Die Faktoren „Alter“ und „Erfahrung“ wurde als stetige Grösse verrechnet. Die Faktoren „Geschlecht", „Stadt“, „Ausbildung“ und „Pensum“ wurden, wie im Originalfragebogen, mit zwei bzw. drei Kategorien in die Analyse eingeschlossen. Eine Übersicht über Definition und Ausprägung der getesteten Einflussfaktoren auf den Fortbildungsbedarf findet sich in Tabelle 1. Als Signifikanzniveau wurde ein p-Wert von 0.05 festgelegt.

\section{Ü1 Resultate}

\section{Ü2 Rücklauf}

„Pilotierungsphase“: Am Lehrärztetreffen wurden 39 Fragebogen abgegeben bzw. innerhalb von 2 Wochen zurückgeschickt.

Definitiver Versand: Der Fragebogen wurde erfolgreich an 1212 Hausärzte im Kanton Zürich verschickt, zusätzlich wurden von der Post 77 Briefe als unzustellbar retourniert. Von den 1212 antworteten 502, der Rücklauf betrug somit 41.4\%. Von diesen 502 gaben 28 an, nicht als Hausarzt tätig zu sein und 13 schickten die Rückmeldung, sie seien bereits pensioniert. Somit konnten wir 461 ausgefüllte Fragebogen in die Analyse einschliessen.

Wie in Abbildung $\mathbf{1}$ ersichtlich, schlossen wir sowohl die Fragebogen der Pilotierungsphase wie auch diejenigen des definitiven Versandes in die Gesamtanalyse ein, woraus sich ein Total von 500 Fragebogen ergab.

\section{Ü2 Demographische Daten}

350 (70.0\%) der Hausärzte waren männlich, 150 (30.0\%) weiblich. Das Durchschnittsalter der Hausärzte betrug 54.1 Jahre (SD 9.0) mit einer Bandbreite zwischen 31 und 82 Jahren. Die Anzahl Jahre seit der Praxiseröffnung reichte von 0 bis 54 Jahren mit einem Durchschnitt von 17.1 Jahren (SD 10.2). 347 (69.4\%) der Praxisstandorte wurden als „städtisch“ klassifiziert, 152 (30.4\%) als „ändlich“. 50 (10.0\%) der Hausärzte arbeiteten mit einem Pensum von weniger als 50\%, 135 (27.0\%) mit einem Pensum von 50-80\% und 311 (62.2\%) mit einem Pensum von über $80 \%$. 


\section{Ü2 Fortbildung und dermatologische Kleinchirurgie in der Praxis}

167 (33.4\%) Hausärzte gaben an, sich während der Weiter- oder Fortbildung vertieft mit Dermatologie beschäftigt zu haben, 330 (66.0\%) verneinten dies. 296 (59.2\%) Hausärzte bejahten, in ihrer Praxis prinzipiell dermatologische Kleinchirurgie durchzuführen, 201 (40.2\%) verneinten dies.

\section{Ü2 Häufigkeit im Praxisalltag}

$116(23.2 \%)$ aller Hausärzte gaben an, täglich oder mindestens einmal pro Woche Patienten mit malignomverdächtigen Hautläsionen in der Praxis zu sehen. Weitere 174 (34.8\%) Hausärzte gaben an, mindestens einmal pro Monat entsprechende Patienten zu sehen. Insgesamt sahen somit 290 (58.0\%) aller Hausärzte wenigstens einmal im Monat Patienten mit malignomverdächtigen Hautläsionen. 198 (39.6\%) Hausärzte sahen weniger als einmal pro Monat entsprechende Patienten in ihrer Praxis.

\section{Ü2 Diagnosesicherheit und Fortbildungsbedarf}

Wie in Abbildung 2 gezeigt, fühlten sich 5 (1.0\%) Hausärzte in der Beurteilung von malignomverdächtigen Hautveränderungen „sehr sicher“, 269 (53.8\%) fühlten sich „eher sicher“, 199 (39.8\%) fühlten sich „eher unsicher“ und 23 (4.6\%) fühlten sich „sehr unsicher“. 294 (58.8\%) Hausärzte gaben an, dass innen die Diagnostik eines Melanoms Mühe bereite, 220 (44.0\%) bekundeten Mühe mit der Diagnostik eines Spinozellulären Karzinoms und 131 (26.2\%) mit der Diagnostik eines Basalzellkarzinoms. Wie in Abbildung 3 gezeigt, bestand für 395 (79.0\%) Hausärzte gemäss unserer Umfrage ein Bedarf an Fortbildung zum Thema Hautkrebs (159 (31.8\%) gaben die Antwort „ja“, 236 (47.5\%) die Antwort „eher ja“). 94 (18.8\%) Hausärzte hatten keinen Bedarf an Fortbildung zum Thema Hautkrebs.

\section{Ü2 Umgang mit malignomverdächtigen Hautveränderungen}

Bei Patienten, die mit malignomverdächtigen Hautveränderungen in die Praxis kamen, untersuchten 50 (10.0\%) Hausärzte nur die Einzelläsion. 196 (39.2\%) Hausärzte gaben an, sowohl die Einzelläsion zu untersuchen wie auch gleichzeitig eine Ganzkörperuntersuchung durchzuführen. 226 (45.2\%) Hausärzte gaben an, solche Patienten nicht selber zu untersuchen, sondern direkt einem Dermatologen zu überweisen. Von den 246 Hausärzten, die malignomverdächtige Hautveränderungen ihrer Patienten selber untersuchten, exzidierten 157 (63.8\%) die Läsion bei entsprechenden Indikation selber, 63 (25.6\%) wiesen den Patienten zur Exzision an einen Dermatologen oder Chirurgen weiter. 


\section{Ü2 Einflussfaktoren auf den Fortbildungsbedarf}

In der Korrelationsanalyse zeigten sich, wie in Tabelle 2 ersichtlich, auch nach Bonferroni-Korrektur zahlreiche statistisch signifikante, jedoch schwache Korrelationen zwischen den untersuchten Einflussfaktoren. Unter anderem zeigten sich positive Korrelationen zwischen dem Faktor „Sicherheit“ und den Faktoren „Alter“, „Erfahrung“, „Pensum“ „Ausbildung“ und „Häufigkeit“. Städtische Praxisstandorte waren signifikant weniger häufig mit dermatologischen Problemen konfrontiert im Vergleich zu ländlichen Praxisstandorten. Städtischer Praxisstandort und subjektive diagnostische Sicherheit waren unabhängige und signifikante Faktoren, welche mit einem verminderten Fortbildungsbedarf assoziiert waren (adjustierte OR 0.42 bzw. 0.40). Ein erhöhter Fortbildungsbedarf zeigte sich bei einem Arbeitspensum von mehr als 50\% (Tabelle 3).

\section{Ü1 Diskussion}

\section{Ü2 Wichtigste Erkenntnisse}

Bei fast $80 \%$ der befragten Hausärzte bestand ein Fortbildungsbedarf zum Thema Hautkrebs. Dieser Fortbildungsbedarf wurde beeinflusst von der subjektiv wahrgenommenen Diagnosesicherheit, vom Praxisstandort und vom Arbeitspensum: Hausärzte mit Praxen in der Stadt und Hausärzte mit hoher subjektiver Diagnosesicherheit hatten weniger Fortbildungsbedarf zum Thema Hautkrebs, Hausärzte mit höherem Arbeitspensum hatten einen höheren Fortbildungsbedarf.

\section{Ü2 Häufigkeit von Hautkrebs im hausärztlichen Alltag, Diagnosesicherheit und} Fortbildungsbedarf

Über die Hälfte der Hausärzte sahen in ihrer Sprechstunde mindestens monatlich Patienten mit malignomverdächtigen Hautläsionen, rund ein Viertel sogar wöchentlich. In einer früheren Schweizer Studie (13) wurde gezeigt, dass das Thema Haut/Hautkrankheiten der fünfthäufigste Konsultationsgrund darstellte nach Konsultationen aufgrund muskuloskeletaler, kardiovaskulärer, respiratorischer und endokriner Ursachen. Eine Studie aus Schottland fand Hautkrankheiten in knapp 9\% der hausärztlichen Konsultationen, über 11\% davon betrafen Hauttumoren (14).

Obwohl sich mehr als die Hälfte der befragten Hausärzte in der Beurteilung von malignomverdächtigen Hautveränderungen sicher fühlten, gaben doch fast $80 \%$ an, einen Bedarf an Fortbildung zum Thema Hautkrebs zu haben. Insbesondere die 
Diagnose des Melanoms bereitete fast $60 \%$ der Befragten Mühe. Bis anhin war es für Hausärzte sehr schwierig, sich im Rahmen ihrer Assistenzzeit dermatologisch fundiert weiterzubilden, da es kaum Stellen auf der Dermatologie gab, die an angehende Hausärzte vergeben wurden. Dies zeigte sich auch darin, dass nur rund ein Drittel der befragten Hausärzte angab, sich während der Weiter- und Fortbildung vertieft mit Dermatologie beschäftig zu haben.

Mit den neuen Hausärzte-Curricula (15) erhalten zumindest einzelne angehende Hausärzte neu die Möglichkeit, halbjährliche Rotationen auf der Dermatologie zu verbringen und sich so dermatologisch fundiert weiterzubilden. Dieses Angebot scheint einem grossen Bedürfnis gerecht zu werden, sind diese Rotationsstellen doch bereits auf Jahre hinaus vergeben.

Unsere Studie dokumentierte ein deutliches Missverhältnis zwischen dem häufigen Vorkommen des Themas in der hausärztlichen Sprechstunde und den bis anhin nur begrenzten dermatologischen Weiter- und Fortbildungsmöglichkeiten. Dieses Missverhältnis wird in Zukunft zwar mit der Etablierung von dermatologischen Rotationsstellen für angehende Hausärzte etwas entschärft, für bereits praktizierende Hausärzte sollten aber vermehrt Fortbildungen zu den Themen Hautkrebsdiagnostik und Management von malignomverdächtigen Hautveränderungen angeboten werden.

\section{Ü2 Einflussfaktoren auf den Fortbildungsbedarf}

Aus der Korrelationsanalyse war ersichtlich, dass ältere Hausärzte mit steigenden Jahren Praxiserfahrung, höherem Pensum, zusätzlicher dermatologischer Weiter/Fortbildung und mit grösserer Häufigkeit des Themas in der Sprechstunde ihre subjektive Diagnosesicherheit höher einschätzten. Hausärzte in der Stadt sahen seltener Patienten mit krebsverdächtigen Hautveränderungen. Mit steigenden Jahren Praxiserfahrung stieg auch der Anteil der Hausärzte mit zusätzlicher dermatologischer Weiter-/Fortbildung.

In der multiplen logistischen Regressionsanalyse zeigte sich, dass der Praxisstandort Stadt vergesellschaftet war mit geringerem Fortbildungsbedarf. Da in der Stadt die Spezialisten-Dichte in der Regel höher ist als auf dem Land, bestand bei den Hausärzten möglicherweise ein niederschwelligeres Überweisungsverhalten, bzw. mehr Patienten suchten mit Hautveränderungen direkt den Dermatologen auf. Somit war die relative Häufigkeit des Themas bei Hausärzten in der Stadt geringer, was zu 
einer geringeren Relevanz in der Sprechstunde und somit zu geringerem Fortbildungsbedarf führte.

Ein höheres Arbeitspensum war tendenziell assoziiert mit erhöhten Fortbildungsbedarf, dies liesse sich möglicherweise damit erklären, dass für Hausärzte mit einem kleinen Arbeitspensum das Thema in ihrer Sprechstunde seltener war und somit von geringerer Relevanz als bei Hausärzten mit höherem Arbeitspensum. Zudem war es denkbar, dass bei reduziertem Pensum die ebenfalls reduzierte Kapazität für Fortbildungen eher für Themen eingesetzt wurde, die im Alltag relevanter und häufiger anzutreffen waren.

Zudem zeigte sich, dass mit zunehmender subjektiver Diagnosesicherheit der Fortbildungsbedarf geringer war. Bei zeitlich limitierten Ressourcen für die Fortbildung war die subjektive Sicherheit im Umgang mit einem medizinischen Problem möglicherweise ein Kriterium, wonach der Bedarf an Fortbildungsangeboten priorisiert wurde. Der Fortbildungs-Effekt war allerdings bei Themen, welche von Hausärzten nicht priorisiert wurden grösser, im Vergleich zu bevorzugt gewählten Themen, wie eine Studie von Sibley et al. (16) zeigte. Ob der geringere Fortbildungsbedarf in unserer Stichprobe Ausdruck eines „Vermeidungsverhaltens“ war, oder ob tatsächlich grössere Sicherheit im Umgang mit malignomverdächtigen Hautveränderungen den Fortbildungsbedarf verminderte, liess sich in unserer Querschnittsanalyse nicht abschliessend beurteilen.

\section{Ü2 Stärken und Schwächen}

Der Rücklauf unsere Studie war mit $41.4 \%$ höher als in der Regel bei einer hausärztliche Umfrage erwartet werden kann (17). Aufgrund der relativ zahlreichen unzustellbaren Briefe musste aber davon ausgegangen werden, dass wir mit unserem Versand nicht alle Hausärzte im Kanton Zürich erreicht hatten. Zudem muss einschränkend betont werden, dass keine objektive Messung von beispielsweise Diagnosesicherheit und Fortbildungsbedarf durchgeführt wurde, sondern dass es sich um eine Selbsteinschätzung der Teilnehmer handelte.

\section{Ü1 Schlussfolgerungen}

Es konnte gezeigt werden, dass Zürcher Hausärzte relativ häufig mit malignomverdächtigen Hautveränderungen ihrer Patienten konfrontiert wurden und 
dass trotz relativ hoher subjektiver Diagnosesicherheit ein deutlicher Fortbildungsbedarf zum Thema Hautkrebs bestand.

\section{Ü1 Dank und Finanzierung}

Wir danken allen Hausärzten, die an der Umfrage teilgenommen haben. Die Studie wurde im Rahmen des Projektes „minSKIN“ von der RRMA finanziert.

\section{Ü1 Anmerkung}

Der Original-Fragebogen ist der Online-Version dieses Artikels beigefügt und kann bei der korrespondierenden Autorin bestellt werden.

\section{Key messages}

- Fast $55 \%$ der befragten Hausärzte fühlten sich sicher in der HautkrebsDiagnostik.

- Dennoch gaben fast $80 \%$ der Hausärzte an, einen Fortbildungsbedarf zum Thema Hautkrebs zu haben.

- Hohe subjektive Diagnosesicherheit und der Praxisstandort Stadt waren assoziiert mit einem geringeren Fortbildungsbedarf, ein höheres Arbeitspensum war assoziiert mit einem höheren Fortbildungsbedarf.

- Hausärzte auf den Land und Hausärzte mit höherem Arbeitspensum hatten wahrscheinlich einen höheren Fortbildungsbedarf, weil sie häufiger Patienten mit malignomverdächtigen Hautveränderungen in ihrer Sprechstunde sahen. 


\section{Referenzen}

1. Cancer in Switzerland. Situation and Development from 1983 up to 2007. http://www.nicer.org/Editor/files/Krebs_in_der_Schweiz_e_web.pdf [database on the Internet]2011. Available from: www.nicer-swiss.ch.

2. Dummer R, Panizzon R, Bloch PH, Burg G. Updated Swiss guidelines for the treatment and follow-up of cutaneous melanoma. Dermatology. 2005;210(1):39-44.

3. Dummer R, Bosch U, Panizzon R, Bloch PH, Burg G. Swiss guidelines for the treatment and follow-up of cutaneous melanoma. Dermatology. 2001;203(1):75-80.

4. Dummer R, Guggenheim M, Arnold AW, Braun R, von Moos R. Updated Swiss guidelines for the treatment and follow-up of cutaneous melanoma. Swiss Med Wkly. 2011;141:w13320.

5. Lutz JM, Francisci S, Mugno E et al. Cancer prevalence in Central Europe: the EUROPREVAL Study. Ann Oncol. 2003 Feb;14(2):313-22.

6. Gandini S, Sera F, Cattaruzza MS et al. Meta-analysis of risk factors for cutaneous melanoma: I. Common and atypical naevi. European Journal of Cancer. 2005;41(1):28 - 44.

7. Gandini S, Sera F, Cattaruzza MS et al. Meta-analysis of risk factors for cutaneous melanoma: II. Sun exposure. Eur J Cancer. 2005 January;41(1):45-60.

8. Gandini S, Sera F, Cattaruzza MS et al. Meta-analysis of risk factors for cutaneous melanoma: III. Family history, actinic damage and phenotypic factors. European Journal of Cancer. 2005;41(14):2040 - 59.

9. Markovic SN, Erickson LA, Rao RD et al. Malignant Melanoma in the 21st Century, Part 1: Epidemiology, Risk Factors, Screening, Prevention, and Diagnosis. Mayo Clinic Proceedings. [Article]. 2007;82(3):364-80.

10. Bentham G, Aase A. Incidence of malignant melanoma of the skin in Norway, 1955-1989: associations with solar ultraviolet radiation, income and holidays abroad. Int J Epidemiol. 1996 Dec;25(6):1132-8.

11. Stang A, Stausberg J, Boedeker W, Kerek-Bodden H, Jockel KH. Nationwide hospitalization costs of skin melanoma and non-melanoma skin cancer in Germany. J Eur Acad Dermatol Venereol. 2008 Jan;22(1):65-72.

12. Badertscher N, Rosemann $T$, Tandjung R, Braun RP. minSKIN does a multifaceted intervention improve the competence in the diagnosis of skin cancer by general practitioners? Study protocol for a randomised controlled trial. Trials. 2011;12:165.

13. Chmiel C, Bhend H, Senn O, Zoller M, Rosemann T. The FIRE project: a milestone for research in primary care in Switzerland. Swiss Med Wkly. 2011;140:w13142.

14. Kerr OA, Tidman MJ, Walker JJ, Aldridge RD, Benton EC. The profile of dermatological problems in primary care. Clinical and Experimental Dermatology. 2010;35(4):380-3.

15. Rosemann T, Bandi-Ott E, Tandjung R. Das Curriculum für Hausarztmedizin am USZ - eine Erfolgsgeschichte. Schweizerische Ärztezeitung. 2012;93(39).

16. Sibley JC, Sackett DL, Neufeld V, Gerrard B, Rudnick KV, Fraser W. A randomized trial of continuing medical education. $N$ Engl J Med. 1982 Mar 4;306(9):511-5.

17. McAvoy BR, Kaner EF. General practice postal surveys: a questionnaire too far? BMJ. 1996 Sep 21;313(7059):732-3; discussion 3-4. 


\section{Tabellen und Abbildungen}

\begin{tabular}{|l|l|}
\hline Geschlecht & Geschlecht: männlich=0, weiblich=1 \\
\hline Alter & Alter in Jahren \\
\hline Erfahrung & Anzahl Jahre Praxiserfahrung \\
\hline Stadt & Praxisstandort: Land=0, Stadt=1 \\
\hline Pensum & Arbeitspensum: $<50 \%=1,50-80 \%=2,>80 \%=3$ \\
\hline Ausbildung & vertiefte dermatol. Weiter-/ Fortbildung: nein=0, ja=1 \\
\hline Häufigkeit & $\begin{array}{l}\text { Häufigkeit des Themas in der Sprechstunde: } \\
\text { monatlich/seltener=0, täglich/wöchentlich=1 }\end{array}$ \\
\hline Sicherheit & $\begin{array}{l}\text { subjektive Diagnosesicherheit: } \\
\text { eher/sehr unsicher=0, eher/sehr sicher=1 }\end{array}$ \\
\hline
\end{tabular}

Tabelle 1: Definition und Ausprägung der möglichen Einflussfaktoren auf den Fortbildungsbedarf. 


\begin{tabular}{|c|c|c|c|c|c|c|c|c|}
\hline & Geschl. & Alter & Erfahrung & Stadt & Pensum & Ausbildung & Häufigkeit & Sicherheit \\
\hline Geschlecht & 1.00 & & & & & & & \\
\hline Alter & $-0.25^{\star \#}$ & 1.00 & & & & & & \\
\hline Erfahrung & $-0.26^{* \#}$ & $0.89^{\star \#}$ & 1.00 & & & & & \\
\hline Stadt & $0.16^{\star \#}$ & -0.03 & -0.03 & 1.00 & & & & \\
\hline Pensum & $-0.46^{\star \#}$ & $0.11^{*}$ & 0.08 & -0.09 & 1.00 & & & \\
\hline Ausbildung & -0.04 & $0.13^{*}$ & $0.16^{\star \#}$ & -0.06 & 0.08 & 1.00 & & \\
\hline Häufigkeit & $-0.21^{* \#}$ & -0.01 & 0.03 & $-0.15^{* \#}$ & $0.18^{\star \#}$ & $0.10^{*}$ & 1.00 & \\
\hline Sicherheit & $-0.21^{\text {*\# }}$ & $0.21^{* \#}$ & $0.24^{\star \#}$ & -0.09 & $0.24^{\star \#}$ & $0.29^{\star \#}$ & $0.22^{\star \#}$ & 1.00 \\
\hline
\end{tabular}

Tabelle 2: Spearman-Korrelationskoeffizienten für die untersuchten Variablen. * $p<0.05$ ohne Bonferroni-Korrektur; ${ }^{*} p<0.05$ nach Bonferroni-Korrektur 


\begin{tabular}{l|ll|ll}
\hline FB-Bedarf & $\begin{array}{l}\text { OR } \\
\text { (unadjustiert) }\end{array}$ & p-Wert & $\begin{array}{l}\text { OR } \\
\text { (adjustiert) }\end{array}$ & p-Wert \\
\hline Geschlecht (Ref.=männlich) & 1.14 & 0.605 & 1.13 & 0.713 \\
Alter (Jahre) & 0.98 & 0.100 & 1.02 & 0.597 \\
Erfahrung & 0.98 & 0.108 & 0.97 & 0.291 \\
Stadt (Ref.=Land) & 0.44 & $\mathbf{0 . 0 0 4}$ & 0.42 & $\mathbf{0 . 0 0 6}$ \\
Pensum 50-80\% (Ref. $<50 \%)$ & 2.20 & $\mathbf{0 . 0 4 8}$ & 2.84 & $\mathbf{0 . 0 1 9}$ \\
Pensum >80\% (Ref. $<50 \%)$ & 1.67 & 0.138 & 2.32 & 0.050 \\
Ausbildung & 1.10 & 0.699 & 1.38 & 0.240 \\
Häufigkeit (Ref.=monatlich/seltener) & 1.04 & 0.889 & 1.09 & 0.784 \\
Sicherheit (Ref.=eher/sehr unsicher) & 0.45 & $\mathbf{0 . 0 0 3}$ & 0.40 & $\mathbf{0 . 0 0 2}$ \\
\hline
\end{tabular}

Tabelle 3: Unadjustierte und adjustierte Odds Ratios (OR) resultierend aus den bivariaten und multiplen logistischen Regressionsanalysen: Auch nach Korrektur blieb im Modell der Fortbildungsbedarf signifikant mit den Faktoren „Stadt“, „Pensum 50$80 \%$ " und „Sicherheit" assoziiert. 


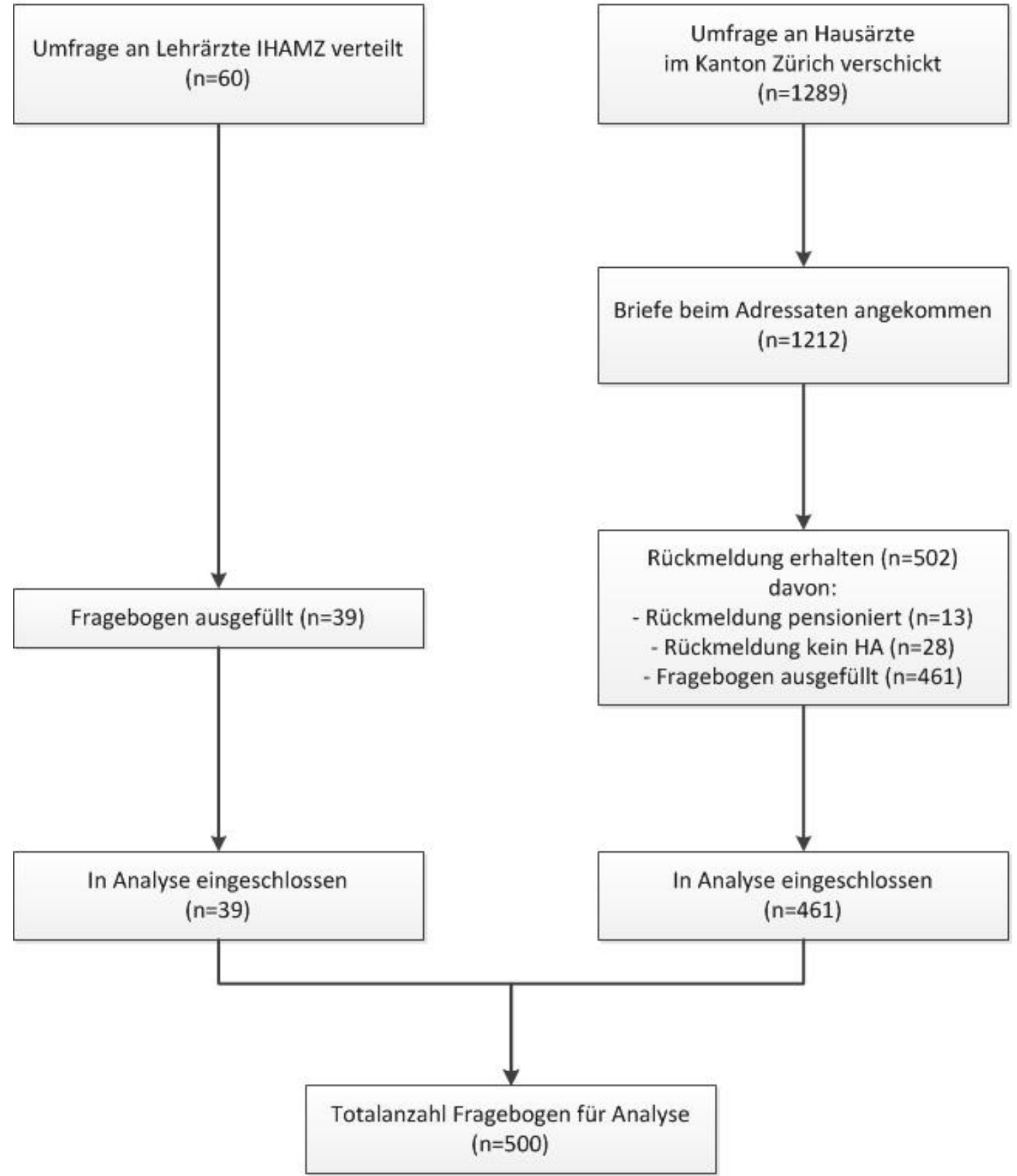

Abbildung 1: Übersicht über die Anzahl verschickter und eingeschlossener Fragebogen 


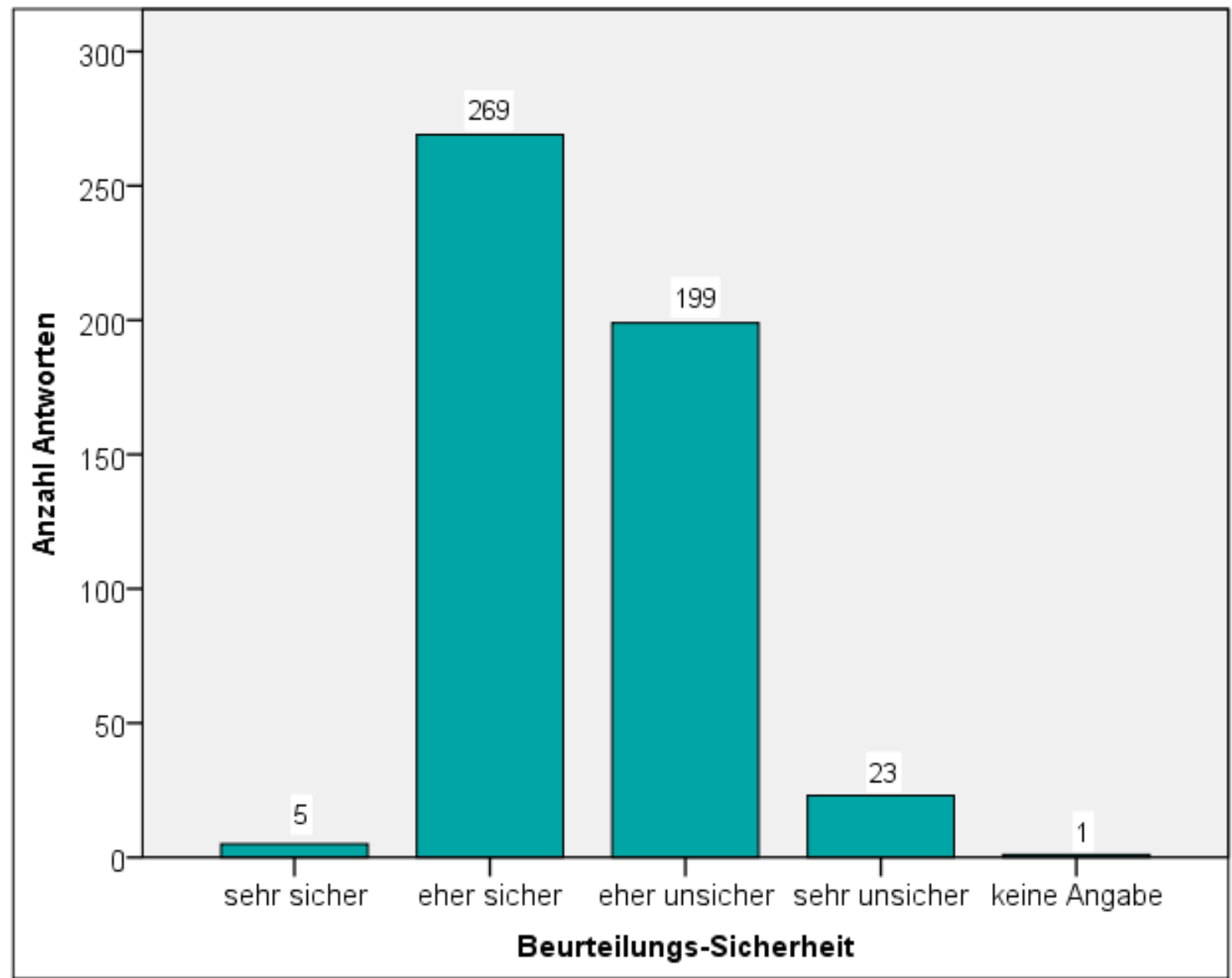

Abbildung 2: Subjektive Einschätzung der Beurteilungs-Sicherheit bei malignomverdächtigen Hautläsionen 


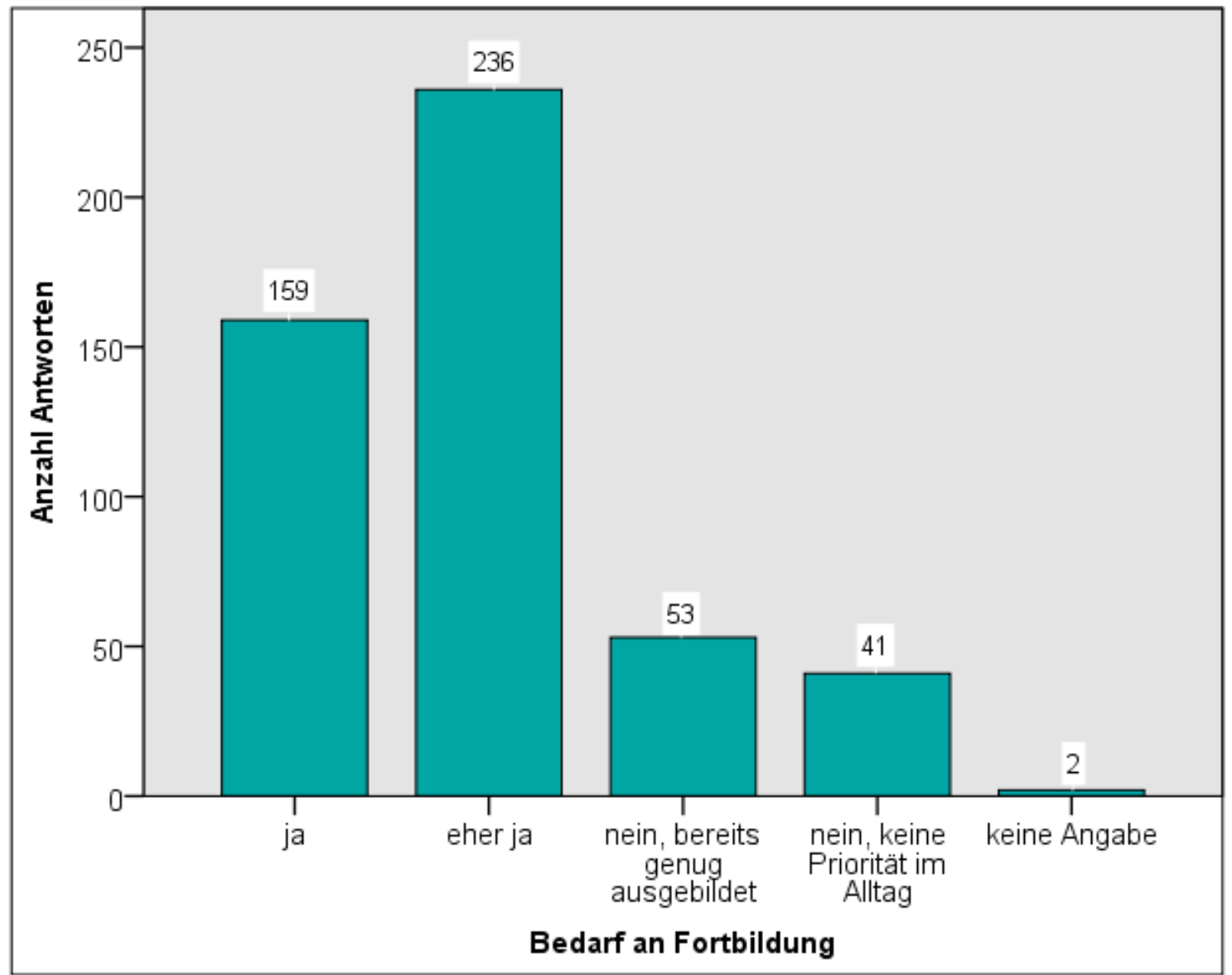

Abbildung 3: Bedarf an Fortbildung zum Thema Hautkrebs 